\title{
¡†rECI\&NE
}

\section{Análisis bibliométrico de la planeación de expansión de los sistemas eléctricos de potencia}

\author{
Bibliometric analysis from expansion planning of electric power systems
}

\author{
Diego Paúl Romero-Carrión' [1; ; Diego Francisco Carrión-Galarza² (1) \\ ${ }^{1}$ Universidad Politécnica Salesiana, Quito, Ecuador, dromeroc4@est.ups.edu.ec \\ ${ }^{2}$ Universidad Politécnica Salesiana, Quito, Ecuador, dcarrion@ups.edu.ec
}

Fecha de recepción: 28 de abril de 2021. Fecha de aprobación: 26 de mayo de 2021

Resumen- Este artículo presenta un análisis bibliométrico sobre la planeación de la expansión de los sistemas eléctricos de potencia, tema de gran importancia debido al continuo crecimiento de la demanda eléctrica. Por tal motivo, los sistemas eléctricos de potencia deben ser diseñados y construidos para prever futuras expansiones mediante el ingreso de nuevas centrales de generación, líneas de transmisión y equipos compensadores. Para el desarrollo de esta investigación se consideran las palabras claves que permitirán encontrar artículos de la misma temática en bases de datos Scopus y Web of Science. Después se organizará esta información de acuerdo con los países, autores, número de citas y organizaciones para hacer una comparación de la información obtenida mediante VOSviewer para indagar criterios de aceptación. Al contar con los artículos seleccionados se realiza la matriz del estado del arte que brindará información sobre formulación, restricción, propuesta y solución al problema.

Palabras clave- Índice de contingencia; metodología de expansión; planeación; transmisión; generación; sistemas eléctricos de potencia.
Abstract- This article presents a bibliometric analysis on the planning of the expansion of electric power systems, a topic of great importance due to the continuous growth of electricity demand. For this reason, electrical power systems must be designed and built to foresee future expansions through the entry of new generation plants, transmission lines and compensating equipment. For the development of this research, keywords that will help find articles on the same subject in Scopus and Web of Science databases will be taken into account. This information will then be organized according to the countries, authors, number of citations and organizations to make a comparison of the information obtained through VOSviewer to inquire about acceptance criteria. By having the selected articles, the matrix of the State of the Art is made which will provide information on formulation, restriction, proposal and solution to the problem

Keywords- Contingency Index; expansion methodology; planning; transmission; generation; electric power systems.

Citar este artículo como: D.P. Romero-Carrión, D.F. Carrión-Galarza, Análisis bibliométrico de la planeación de expansión de los Sistemas Eléctricos de Potencia. ITECKNE, 18 (2), 2021 pp. 150 - 160 DOI: https://doi.org/10.15332/iteckne.v18i2.2610 


\section{INTRODUCCIÓN}

Las centrales de generación y líneas de transmisión $(\mathrm{L} / \mathrm{T})$ necesitan ser repotenciadas para atender el crecimiento de la demanda eléctrica, brindar confiablidad al Sistema Eléctrico de Potencia (SEP). [1] La normativa establece que el SEP deberá abastecer la demanda al fallar un único elemento basado en el criterio ( $\mathrm{N}-1)$.

Garantizar el abastecimiento del suministro de energía eléctrica a los consumidores considerando la proyección de la demanda en un tiempo determinado es importante y para eso se hace el estudio del TEP óptimo y adecuado. [2] El estudio del TEP debe ejecutarse con anterioridad a la construcción y visualizando desde la planificación para la ejecución de acciones a corto, mediano y largo plazo. La solución planteada es el flujo óptimo de potencia CC porque su principal ventaja es solucionar el problema de forma rápida, al compararlo con un flujo óptimo de potencia CA se tiene un mínimo error.

Para la planificación de la expansión de transmisión (TEP) los autores plantean la existencia de inconvenientes, [3] se debe elegir un grupo de circuitos del sistema de transmisión y garantizar confiabilidad en su funcionamiento con una inversión no elevada. Considera la utilización de un algoritmo heurístico constructivo (CHA) para solucionar la planificación en sistemas de transmisión de energía eléctrica.

La problemática de la planificación de la expansión de la generación (GEP) y TEP están relacionadas con el lugar, duración de la construcción, tipo de central, capacidad de generación y construcción de nuevas $L / T$, subestaciones, capacidad [4], para [5] el TEP debe considerar la contingencia (N-k) e incertidumbre de la demanda y la generación eléctrica.

La planificación de la expansión de sistemas de transmisión [6] puede ser dinámica o estática, caracterizadas por ser simples y complejas, respectivamente. La expansión dinámica es basada en la expansión del sistema en un lapso de tiempo y se analiza el comportamiento del sistema en cada fase de planificación; y la expansión estática se plantea como un problema de programación matemática, para así reducir los costos de operación e inversión. El problema del TEP es una labor complicada, es necesario conocer el lugar, el tiempo y las instalaciones por implementar que brinden garantía, confiabilidad al suministrar energía eléctrica.

En [7] se recomienda utilizar un marco iterativo que permita la resolución del problema de optimización de la planificación de la expansión de la capacidad de transmisión (TCEP) estocástica junto con el análisis de contingencia N-1. El método Monte Carlo es utilizado para los diferentes escenarios que se producen en el inicio de la etapa de planificación.

En los SEP [8] el fujo de potencia se considera un estudio importante por realizar, que sirve para la planificación, operación, expansión. Seleccionar la contingencia se desarrolla calculando los siguientes índices de desempeño:
Índice de rendimiento activo (PIP) e índice de rendimiento de potencia reactiva (PIV) al existir una interrupción en una L/T. Se utiliza el método Newton Rhapson para calcular los índices de desempeño PIP, PIV y el software DigSILENT Power Factory para clasificar la contingencia.

De acuerdo con lo descrito anteriormente, se conoce que hay varias investigaciones que tienen como propuesta principal la planeación de la expansión de capacidad de transmisión, al consultar sobre transmisión y generación consideran como herramienta de solución la programación lineal de enteros mixtos (MILP) para la resolución de la problemática de la expansión en sistemas de transmisión de energía eléctrica, para la TEP utilizan programación matemática y métodos heurísticos que son dos enfoques para encontrar soluciones.

La finalidad del artículo es utilizar palabras clave relacionadas con el tema "Planeación de la Expansión del SEP", obtener artículos de revistas de alto impacto relacionadas con la temática y palabras claves, que hayan sido referenciadas y citadas por otros autores; esto servirá para conocer las restricciones y soluciones a los problemas que han sido planteados por los autores. Las bases de datos Scopus y Web of Science serán herramientas que brindarán información del entorno mundial acerca de países e investigadores que han realizado investigaciones similares al tema planteado.

El artículo será estructurado como se detalla a continuación: en la sección II revisión de la planificación de la expansión de la generación y transmisión. En la sección III soluciones para la problemática mediante varias herramientas. En la sección IV diferentes consideraciones de los investigadores y, finalmente, en la sección $\mathrm{V}$ las conclusiones.

\section{DESARROLLO DEL ARTÍCULO}

En los SEP correctamente diseñados y construidos, [9] al originarse una falla en varias $\mathrm{L} / \mathrm{T}$ no provocaría una sobrecarga en otras $\mathrm{L} / \mathrm{T}$ en la totalidad de eventos de la carga y no afectaría las partes factibles, esto es una respuesta adecuada de la problemática de optimización de la planificación de la expansión de la capacidad de transmisión (TCEP), caso contrario se podría detectar una falla y eliminarla de forma segura en el análisis de contingencia.

El continuo aumento de la demanda de energía eléctrica es consecuencia del crecimiento de la población y del sector industrial, provocando que el SEP actual presente falencias para atender la demanda eléctrica y garantizar un alto nivel de seguridad, confiabilidad y calidad. En el futuro se convierte en un verdadero desafío garantizar los parámetros del SEP, [10] sugiere un enfoque computacional efectivo para optimizar la problemática de la planificación del GEP, se considera el MILP de gran escala. La función del GEP y TEP es tener niveles de confiabilidad en proyectar la demanda eléctrica para cumplir con los límites de generación y transmisión. 
La función del GEP y TEP es brindar niveles de confiabilidad en la proyección de la demanda eléctrica, al cumplirse con los límites de generación y transmisión. La problemática del GEP y TEP se soluciona economizando en el plan de expansión y se reflejará en la construcción de sistemas de generación, transmisión o ambos. Realizar una inversión económica no planificada conlleva tener costos elevados y no invertir y a tener un nivel bajo de confiabilidad, el saber combinar estas situaciones es un gran reto para el personal encargado de SEP, así también para diseñadores, planificadores, operadores [11].

En [12] se considera la inestabilidad del voltaje referente a la parte dinámica del SEP y la situación de probabilidad de contingencias, si estas son críticas la forma de hacer un análisis preciso es mediante el flujo de carga en estado estático de perturbaciones para conocer la debilidad del SEP. Sugiere utilizar la matriz jacobiana para un flujo de carga dinámico, donde la determinante más pequeña permite obtener el grado de inestabilidad de voltaje.

Una investigación importante en un SEP que ha sido estudiada con profundidad en los últimos años es la TEP [13]. Para solucionar se han desarrollado algunos modelos de la TEP en los últimos años como programación matemática y métodos heurísticos que son dos herramientas de solución. Para disminuir la sobrecarga en un sistema de transmisión ya construido, es necesario construir un número adecuado de $\mathrm{L} / \mathrm{T}$ para aumentar la eficiencia y confiabilidad de todo el sistema.

La resolución del TEP conjuntamente con el acoplamiento de generación eólica debe solucionarse. Al aumentar su capacidad la fuente de generación hace que las L/T se sobrecarguen, para evitar esta condición se deben construir nuevas $\mathrm{L} / \mathrm{T}$ y dar un nivel de seguridad óptimo, la expansión del sistema de transmisión debe suplir los inconvenientes técnicos, económicos y de seguridad [14]. Los autores consideran un estudio multi etapa para la generar escenarios de TEP y GEP considerando centrales de generación eólica, reduciendo la complejidad del problema de optimización mediante la descomposición de Benders, con lo cual los problemas de la incertidumbre que genera la demanda y la disponibilidad del recurso eólico se minimizan.

EI TEP es parte importante de la planificación del sistema eléctrico, [15] considerando que la generación y selección del sistema de transmisión mantengan la capacidad de transmitir para abastecer la carga, se presenta la resolución de modelos de optimización estocásticos que son parte de la distribución de probabilidades. La simulación es una herramienta importante para la solución de problemas de optimización estocástica para enfoques de simulación-optimización, las variables de forma aleatoria son muestreadas a partir de las propias distribuciones.

En [16] utiliza un método detallado de la planificación conjunta del sistema de transmisión y expansión del sistema de generación del SEP, implementa un modelo de optimización mediante MILP que determinaría el plan de expansión óptimo, considerando la construcción de grandes centrales eléctricas y la generación de energía eléctrica utilizando energías renovables. Investiga la problemática de planificación en la expansión del SEP, la capacidad de generación y el sistema de transmisión de forma individual, con herramienta como el entero mixto y programación no lineal. Utiliza incorporación de incertidumbre, método probabilístico y Monte Carlo.

EI TEP es parte primordial de la planificación del SEP, [17] los niveles adecuados y aceptables de confiabilidad se obtienen al comparar la frecuencia de interrupción real y los índices de duración de interrupciones. DigSILENT es utilizado para el cálculo del análisis de flujo de potencia en estado estable; y valoración de confiabilidad de un sistema de transmisión. Finalmente, la pérdida de un elemento luego de una falla (Contingencia N-1) implica que el sistema debería operar sin existir afectaciones. Hay condiciones normales y de emergencia: las primeras son del tipo térmicas para la L/T, el transformador de potencia y los valores de voltaje tienden a mantenerse de forma indefinida sin riesgo al presentarse una falla, las segundas son del tipo térmica, de voltaje que el equipo debe soportar durante un periodo corto de tiempo.

Contingencia N-1 es caracterizada por la desconexión de un elemento del SEP como un generador, L/T, transformador, etc. [18] El estudio de confiabilidad se resuelve planteando índices de confiabilidad para realizar cuantificaciones de los elementos y equipos del SEP que podrían fallar. La utilización de Conmutación de Transmisión Óptima (OTS) garantiza que no se vera interrumpida la demanda eléctrica, el OTS disminuye el efecto en el SEP por una $\mathrm{L} / \mathrm{T}$ que falle. Al desconectarse las $\mathrm{L} / \mathrm{T}$, el flujo de potencia aumenta y esto provocaría sobrecargas en las L/T.

\section{SOLUCIONES}

Las soluciones encontradas luego de realizar el análisis bibliométrico para la planificación de la expansión del SEP, son las siguientes:

- MILP

EI SEP necesita una adecuada planificación que debe ser realizada a largo plazo, considerando combinar los sistemas de transmisión y generación para abastecer en el futuro. [19] Realizarlo de esta manera permitirá tener una operación confiable y viablemente económica. La solución para este inconveniente del TEP será desarrollada mediante MILP.

\section{- TEP}

Al existir crecimiento de la demanda, [20]this paper presents security-constrained generation and transmission expansion planning (SCGTEP debido al aumento de carga de los usuarios o el ingreso de nuevos usuarios de energía eléctrica, aparecen nuevos retos para los índices operativos del sistema, que se refleja en el crecimiento de las pérdidas de energía, daños en el perfil de voltaje y aparición de sobrecargas. En el 
sistema de transmisión se debe considerar que el índice de seguridad también sufre afectaciones reflejados en el aumento de carga que minimiza la estabilidad del voltaje estático. Al presentarse deslastre de carga o confiabilidad (Contingencia $\mathrm{N}-1$ ) se considera el método TGEP.

Para el TEP en multi etapas, [21] es necesario conocer el lugar, tiempo y la clase de refuerzos que deberán instalarse en el SEP para que abastezca la demanda eléctrica y brinde confiabilidad. Se consideran las pérdidas óhmicas que incluyen los costos operativos para el procedimiento de optimización; se usará el modelo del flujo de potencia CC, las pérdidas se obtienen con el cálculo del vector del ángulo del voltaje al resolver la programación lineal en una configuración escogida. Las pérdidas son repartidas como cargas, las barras (terminales $\mathrm{i}, \mathrm{j}$ ) obtienen el $50 \%$ de las pérdidas óhmicas del sistema conectado.

Para resolver el TEP se plantean las metodologías metaheurística y matemática [22] como métodos de optimización metaheurística que abarcan la optimización de colonia de hormigas, estrategia evolutiva, algoritmos de abejas melíferas, programación evolutiva, etc.; y métodos de optimización matemática, considerándose programación lineal y no lineal, programación de enteros mixtos, algoritmo iterativo, árbol de decisión etc. Cada método tiene sus ventajas y desventajas.

La resolución de TEP se realiza utilizando modelos de optimización matemática, considerado un modelo óptimo, porque no abarca temas técnicos, medio ambientales y económicos. [23] La solución para el análisis e investigación de sistemas de transmisión eléctrica es el flujo de potencia, empleado en estado estacionario para obtener la magnitud de voltaje, corriente en cada L/T, barras. Los flujos de potencia permiten analizar las situaciones que puedan ocurrir en el SEP y se plantean los flujos de potencia CC y CA. El flujo de potencia CC analiza únicamente la potencia activa sin analizar potencia reactiva y pérdidas de potencia. El flujo de potencia CA es más complejo, sin embargo abarca la potencia activa, reactiva durante la planificación garantizando estabilidad del SEP y para la optimización del TEP analiza mayores parámetros y restricciones.

Para la planificación de la expansión de las redes de transmisión (TNEP), [24] propone una solución matemática correspondiente a un modelo de flujo de potencia CC y plantea únicamente balancear la potencia activa del sistema sin considerar las pérdidas. Se trata de una programación no lineal de enteros mixtos (MINLP).

La problemática del TNEP se soluciona con un modelo del flujo de potencia CC, [25] uniendo el ángulo de voltaje y la potencia activa, obteniendo el flujo de potencia del sistema de transmisión con facilidad, mínima complicación computacional y adecuada precisión.

\section{- GEP}

Al plantear una confiabilidad N-1 el SEP debe considerarse durable e idónea para soportar una falla en cualquier elemento con probabilidad alta. [26] La confiabilidad contiene varias reglas, el conocer la situación y la respuesta del operador al ocurrir estos sucesos. Los costos referidos a generación simulan el acuerdo de la unidad y el despacho, considerando estudios económicos para propuestas de expansión en sistemas de transmisión. El tener potencias grandes que provienen de energía solar y eólica junto con el almacenamiento y la demanda apreciable hacen que el enfoque deba cambiar.

La expansión de la capacidad de generación [27] propone un modelo novedoso, fundamentando la inversión en unidades de generación. Presenta un modelo de expansión sobre la capacidad de generación, teniendo en cuenta rampas de potencia, potencia mínima de salida, subida y bajada de tiempos.

La planificación de expansión de la capacidad (CEP) [28] fue planteada como un inconveniente de optimización por primera ocasión en 1957 luego de desarrollar técnicas computacionales y de descomposición, se obtiene el CEP en modelos simples de SEP reales. La problemática del CEP se desarrolla de forma individual con inversión en transmisión y generación porque cada sistema tiene diferente tipo de infraestructura.

Realiza dos enfoques [29]. El microenfoque se basa en métodos analíticos sofisticados de investigación operativa y metaheurística para solucionar la complejidad en restricciones de transmisión no lineal y la confiabilidad. El macroenfoque establece la información del GEP detallado que tiene una restricción y no garantiza la optimización global. Se considera que el microenfoque abarca una problemática involucrada en un mayor marco macroeconómico que formula planificar el sistema económico, energético en función a multisectores.

Se hace con una planificación centralizada investigando el rendimiento del método de reducción de escenario. [30] El GEP tradicional busca hallar tecnologías ideales, tamaño de expansión y construir una nueva unidad de generación disminuyendo costos; y garantizando que la capacidad sea suficiente para satisfacer el aumento de la demanda proyectada. Plantea un método heurístico con reducción de escenarios para la planificación del GEP para un largo plazo.

Demuestra un modelo dinámico del GEP utilizando el cálculo de la probabilidad de pérdida de carga que es aplicado como criterio de confiabilidad. Considera un GEP estático y dinámico [31]. El estático es de único período y tiene menor complejidad computacional ante el problema y en el dinámico la problemática de planificación de períodos múltiples con una mayor complejidad computacional. 
Los Créditos de Energía Renovable (REC) son considerados para crear normativas en el mercado renovable, algo común en EE.UU y que ha servido para dar incentivos en la implementación de nuevas centrales eléctricas. Una solución del GEP aprovechando energía renovable en el SEP [32] es mediante el método del algoritmo iterativo de punto fijo y método de Karush - Kuhn - Tucker (KKT) utilizado para resolver problemas de programación matemática.

\section{- Metodología heurística}

Para encontrar una solución a la planificación de los sistemas de transmisión [33]the expansion decision (0 or 1 se realizan modelos matemáticos (descomposición), algoritmos (combinatoria) y métodos (heurística) e indica que: La descomposición no se recomienda aplicar a SEP de gran escala. La combinatoria encuentran solución de gran calidad y es necesario utilizar un buen PC para la cantidad de parámetros por solucionar. La heurística tiene facilidad de implementación, es fuerte y requiere un esfuerzo computacional pequeño.

La representación del sistema de transmisión es mediante modelos matemáticos simples para la planificación de la expansión. [34] hace un análisis de un sistema de transmisión estático y centralizado con criterios de seguridad, utilizando un modelo de flujo de potencia CC. Considera la contingencia ( $\mathrm{N}-1$ ) para establecer que al existir una falla en alguna $\mathrm{L} / \mathrm{T}$, el resto del sistema de transmisión funcionará correctamente. Proponen dos tipos de soluciones: La planificación tradicional mediante modelo matemático y heurístico de dos fases. La utilización de metaheurísticas mediante algoritmos heurísticos y técnicas de optimización.

La planificación con restricciones de seguridad basada en las propuestas tradicional y metaheurística. [34] La tradicional considera un modelo matemático mediante un software comercial que soluciona el problema y da un enfoque heurístico. La metodología utilizada por los autores para resolver el modelo matemático es mediante un algoritmo metaheurístico.

\section{- Algoritmo estocástico}

Es fundamental que el SEP brinde seguridad y confiabilidad ante la existencia de fallas o eventos imprevistos, por esta situación se considera la contingencia ( $\mathrm{N}-1)$. En [35]a bi-objective robust model is proposed for network expansion planning (NEP la planificación de la expansión se compara con los resultados obtenidos de los métodos robusto y estocástico, los autores indican que del método robusto se obtiene una solución de mayor eficiencia en comparación con el modelo estocástico, porque el método robusto abarca la contingencia más severa de un solo elemento del sistema y ante la ocurrencia debe garantizarse la seguridad.

El planteamiento para solucionar incertidumbres en la expansión de la transmisión es: [36] La optimización estocástica considerando incertidumbres aleatorias de forma directa, y la programación restringida al azar buscando analizar el peor de los eventos por ocurrir para minimizar el impacto. También informa que las incertidumbres son ignoradas por los investigadores, por eso requieren un mayor estudio sobre expansión y costos de generación.

\section{- Descomposición de Benders}

La gran cantidad de avances en el análisis de contingencia en los últimos 10 años dejan el camino para estudios más avanzados y profundos. [37] La planificación de la expansión de la generación y transmisión (TGEP) se realizará considerando restricciones de contingencia. Pretende el diseño y aumento del sistema de energía con un costo mínimo para cumplir con las restricciones de contingencia (N-k). El algoritmo propuesto para solucionar la optimización del sistema eléctrico es la descomposición de Benders.

\section{CONSIDERACIONES DE LA INVESTIGACIÓN}

Utilizando el método histórico descriptivo con las siguientes palabras claves: Analysis, contingency index, planning, expansion, Electric Power Systems, se buscó información en documentos del tipo journal desde el 2010 al 2020, procedimiento realizado en las bases de datos Scopus y Web of Science.

Después de hacer una minería de datos, usando el método experimental, se realizó un análisis bibliométrico con ayuda de software VOSviewer. Dicha información permitió realizar una clasificación y un análisis de la información referente a países, autores y cantidad de citas, teniendo en cuenta la relación existente con referencia a la temática que fue descrita y posteriormente se elaboraron tablas y gráficos del tipo red donde se observaron los 10 autores con más relevancia respecto al tema investigado.

Después de obtener la información requerida, se elaboraron tablas de tipo comparativo de los 10 autores y países que cuenten con la mayor cantidad de citas y número de publicaciones desde el 2010 hasta el 2020 respecto al índice de contingencia en la planeación de la expansión de los sistemas eléctricos de potencia.

En la Fig. 1 se observa un diagrama de flujo que contiene información de la metodología aplicada para el análisis bibliométrico para la investigación.

\section{- Análisis de resultados}

Después de obtener los artículos para el estudio de la expansión del SEP, se realizó la clasificación de información, teniendo en cuenta los artículos relevantes, citas, autores y organizaciones por países desde el año 2010 al 2020.

\section{- Análisis bibliométrico}

Se hizo la búsqueda de artículos utilizando las siguientes palabras clave: planning, expansion, Electric Power Systems en las bases de datos Scopus y Web of Science, se obtuvieron 332 y 1048 resultados, respectivamente del tipo macro, los mismos que sirvieron para construir y observar en VOSviewer las redes bibliométricas, que están basadas en las palabras clave y que alrededor de 55 países han re- 
alizado publicaciones sobre el tema expansión, planificación de sistemas eléctricos de potencia y se procedió a escoger a aquellos países que mínimo hayan publicado diez artículos del tema investigado, al hacerlo disminuyó a 41 países y los que tienen mayor número de publicaciones están representados por los nodos más grandes y se visualiza en la Fig. 2.

Fig. 1. METODOLOGÍA UTILIZADA PARA ANÁLISIS BIBLIOMÉTRICO REFERENTE A LA EXPANSIÓN DEL SEP

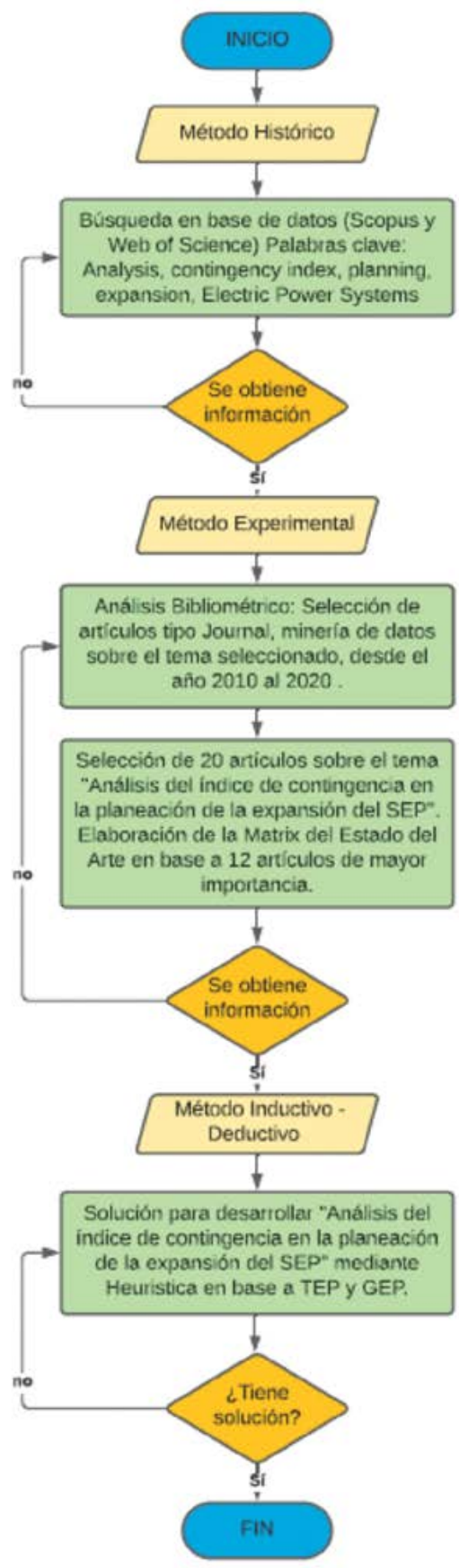

Fig. 2. PAÍSES RELACIONADOS CON LA TEMÁTICA PLANEACIÓN, EXPANSIÓN DEL SEP

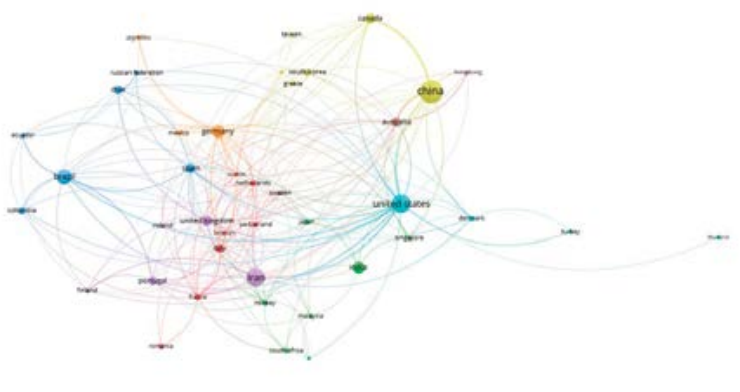

Fuente: Los autores.

De la figura anterior se observa que en la Tabla I, los países China, EE.UU, Irán, Brasil corresponden a los nodos más grandes, basándose en el número de publicaciones; mientras que países como Reino Unido, Canadá están ligados directamente con los países que tienen mayor número de publicaciones.

Tabla I.

PAÍSES CON MAYOR NÚMERO DE PUBLICACIONES REFERENTE A LA PLANEACIÓN, EXPANSIÓN DEL SEP.

\begin{tabular}{|c|c|c|c|}
\hline $\mathbf{N}^{\circ}$ & Países & $\mathbf{N}^{\circ}$ Artículos & Ponderación \\
\hline 1 & China & 400 & $25,39 \%$ \\
\hline 2 & EEUU & 274 & $17,39 \%$ \\
\hline 3 & Irán & 258 & $16,38 \%$ \\
\hline 4 & Brasil & 164 & $10,41 \%$ \\
\hline 5 & Alemania & 123 & $7,81 \%$ \\
\hline 7 & Reino Unido & 86 & $5,46 \%$ \\
\hline 8 & Canadá & 76 & $4,82 \%$ \\
\hline 9 & Australia & 72 & $4,57 \%$ \\
\hline 10 & Italia & 71 & $3,51 \%$ \\
\hline
\end{tabular}

Fuente: Los autores.

Con la información de la Tabla I se hizo un análisis, considerando a los 10 países con el mayor número de publicaciones referentes al tema investigado. La ponderación es el porcentaje de publicaciones realizadas por cada país en relación con la totalidad de 1575 artículos, se obtiene realizando una regla de tres simple y es aplicado en todas las tablas. En la Fig. 3 se observan 10 países que han realizado más publicaciones, siendo China el de mayor cantidad e Italia el de menor cantidad de publicaciones. 
Fig. 3. RANGO DE 10 PAÍSES CON MAYOR NÚMERO DE PUBLICACIONES REFERENTE A LA PLANEACIÓN, EXPANSIÓN DEL SEP

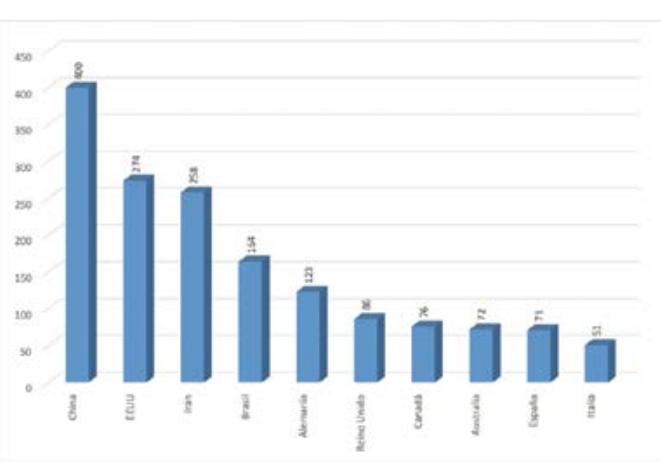

Fuente: Los autores.

En la Tabla II se describen los países con mayor cantidad de citas, en un rango de 10 países, el primero es el más citado y el décimo es el menos citado.

Tabla II.

PAÍSES CON MAYOR NÚMERO DE CITAS REFERENTE A LA PLANEACIÓN, EXPANSIÓN DEL SEP

\begin{tabular}{|c|c|c|c|}
\hline $\mathbf{N}^{\circ}$ & Países & $\mathbf{N}^{\circ}$ Citas & Ponderación \\
\hline 1 & China & 5033 & $21,62 \%$ \\
\hline 2 & EEUU & 4988 & $21,42 \%$ \\
\hline 3 & Irán & 3994 & $17,15 \%$ \\
\hline 4 & Brasil & 1764 & $7,57 \%$ \\
\hline 5 & Reino Unido & 1682 & $7,22 \%$ \\
\hline 6 & España & 1424 & $6,11 \%$ \\
\hline 7 & Australia & 1219 & $5,23 \%$ \\
\hline 8 & Alemania & 1210 & $5,19 \%$ \\
\hline 9 & Canadá & 1101 & $4,73 \%$ \\
\hline 10 & Italia & 864 & $3,71 \%$ \\
\hline
\end{tabular}

Fuente: Los autores.

Fig. 4. RANGO DE 10 PAÍSES CON MAYOR NÚMERO DE CITAS REFERENTE A LA PLANEACIÓN, EXPANSIÓN DEL SEP

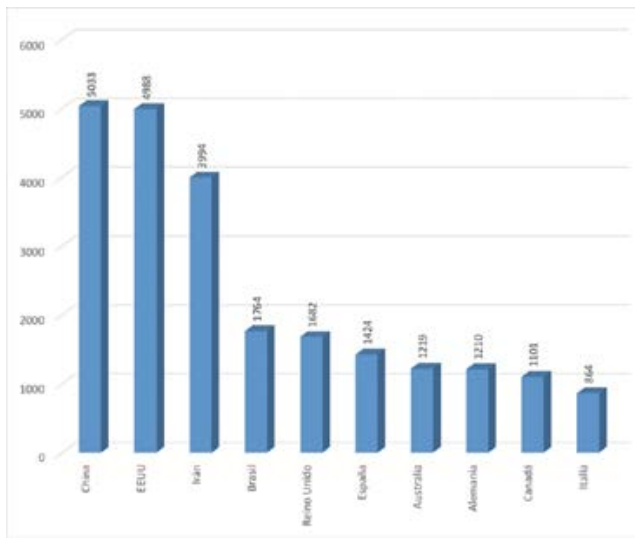

Fuente: Los autores.
Considerando la cantidad de artículos y publicaciones por cada país, se observa que la mayoría son autores chinos, estadounidenses e iraníes y se representa en la Fig. 5.

Fig. 5. AUTORES CON PUBLICACIONES REFERENTES A LA PLANEACIÓN, EXPANSIÓN DEL SEP

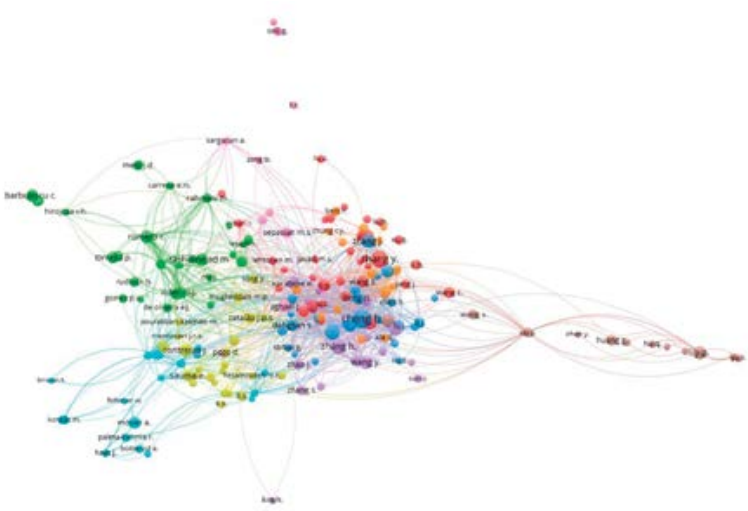

Fuente: Los autores.

La Tabla III contiene información de los autores que tienen mayores citas referentes a publicaciones realizadas sobre la planificación, expansión del SEP.

Tabla III. AUTORES CON MAYOR NÚMERO DE CITAS REFERENTE A LA PLANEACIÓN, EXPANSIÓN DEL SEP

\begin{tabular}{|c|c|c|c|}
\hline $\mathbf{N}^{\circ}$ & Países & $\mathbf{N}^{\circ}$ Citas & Ponderación \\
\hline 1 & Shahidehpour M. & 944 & $16,65 \%$ \\
\hline 2 & Hu Z. & 755 & $13,32 \%$ \\
\hline 3 & Huang G.H. & 627 & $11,06 \%$ \\
\hline 4 & Hemmati R. & 553 & $9,75 \%$ \\
\hline 5 & Zhang X & 525 & $9,26 \%$ \\
\hline 7 & Zhang N & 497 & $8,77 \%$ \\
\hline 8 & Dong Z.Y. & 473 & $7,34 \%$ \\
\hline 9 & Qhodaei A & 444 & $7,50 \%$ \\
\hline 10 & Fotuhi & 424 & $7,48 \%$ \\
\hline
\end{tabular}

Fuente: Los autores.

En la Fig. 6 se visualiza en el rango de 10 autores más citados, el autor Shahidehpour M. es el primero, tiene 944 citas y el autor Fotuhi-Firuzabad M. es el décimo, tiene 424 citas.

En la figura 6, se visualiza que los autores con la mayor cantidad de citas son de nacionalidad china, los autores seleccionados han realizado como mínimo cinco publicaciones. 
Con la información de países, citas, autores se hace el análisis de organizaciones con publicaciones relacionadas con la temática de investigación y se organiza por la cantidad de citas, se representa en la Fig. 7.

Fig. 6. AUTORES CON PUBLICACIONES RELEVANTES REFERENTE A LA PLANEACIÓN, EXPANSIÓN DEL SEP A NIVEL MUNDIAL

Fuente: Los autores.

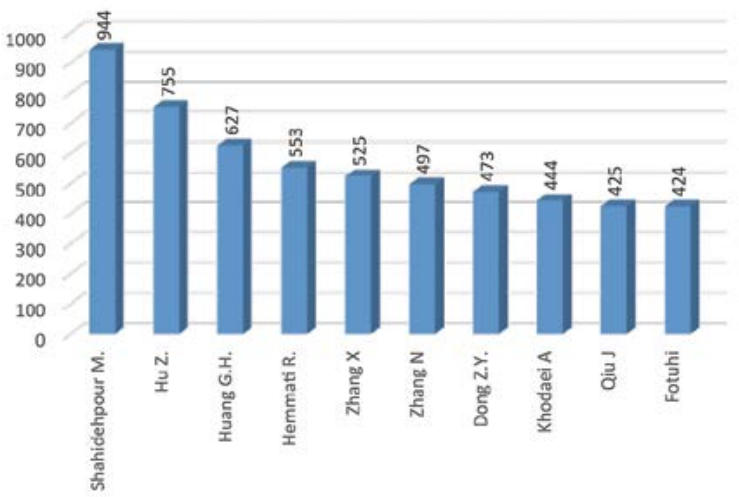

Fig. 7. ORGANIZACIONES CON PUBLICACIONES REFERENTES A LA PLANEACIÓN, EXPANSIÓN DEL SEP

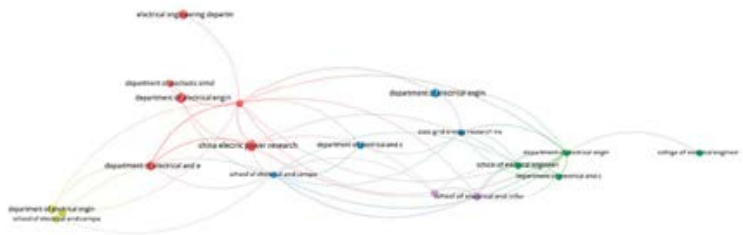

Fuente: Los autores.

La Tabla IV presenta a las organizaciones que tienen mayor cantidad de citas referentes a publicaciones realizadas sobre la planificación y expansión del SEP.

En la Fig. 8 se considera un rango de 10 organizaciones con el mayor número de citas, en el primer lugar se encuentra el Department of Electrical Engineering, University of Isfahan, Isfahan, Iran con 419 citas y en el décimo lugar se encuentra State Key Laboratory of Alternate Electrical Power System with Renewable Energy Sources, North China Electric Power University, Beijing, 102206, China con 96 citas.

En la Fig. 8 se observa que la organización con la mayor cantidad de citas es de Irán con 419 y la que tiene una menor cantidad de citas es de China. Se indica que hay cuatro organizaciones de China, dos organizaciones de Irán y una organización de Alemania, Australia, EE.UU, Grecia, respectivamente.
Tabla IV.

ORGANIZACIONES CON MAYOR NÚMERO DE CITAS EN PUBLICACIONES REFERENTES A LA PLANEACIÓN, EXPANSIÓN DEL SEP

\begin{tabular}{|c|c|c|c|}
\hline $\mathbf{N}^{\circ}$ & Organizaciones & $\mathbf{N}^{\circ}$ Citas & Ponderación \\
\hline 1 & $\begin{array}{l}\text { Department of Electrical } \\
\text { Engineering, University of Isfahan, } \\
\text { Isfahan, Iran. }\end{array}$ & 419 & $22,67 \%$ \\
\hline 2 & $\begin{array}{l}\text { School of Electrical and } \\
\text { Information Engineering, } \\
\text { university of Sydney, Sydney, Nsw, } \\
\text { Australia. }\end{array}$ & 236 & $12,77 \%$ \\
\hline 3 & $\begin{array}{c}\text { School of Electrical and Computer } \\
\text { Engineering, National Technical } \\
\text { University of Athens (ntua), Athens, } \\
\text { Greece. }\end{array}$ & 210 & $11,36 \%$ \\
\hline 4 & $\begin{array}{l}\text { School of Electrical Engineering, } \\
\text { Beijing Jiaotong University, Beijing, } \\
\text { 100044, China. }\end{array}$ & 176 & $9,52 \%$ \\
\hline 5 & $\begin{array}{c}\text { Department of Electrical } \\
\text { Engineering, Tsinghua University, } \\
\text { Beijing, 100084, China }\end{array}$ & 174 & $9,42 \%$ \\
\hline 6 & $\begin{array}{l}\text { Department of Stochastic } \\
\text { Simulation and Safety Research } \\
\text { for Hydrosystems (iws/sc simtech), } \\
\text { University of Stuttgart, Germany. }\end{array}$ & 151 & $8,17 \%$ \\
\hline 7 & $\begin{array}{c}\text { School of Electrical and Computer } \\
\text { Engineering, University of Tehran, } \\
\text { Tehran, Iran. }\end{array}$ & 137 & $7,41 \%$ \\
\hline 8 & $\begin{array}{l}\text { Department of Electrical and } \\
\text { Computer Engineering, lowa State } \\
\text { University, Ames, ia 50011, United } \\
\text { States. }\end{array}$ & 137 & $7,41 \%$ \\
\hline 9 & $\begin{array}{c}\text { State Grid Energy Research } \\
\text { Institute, Beijing, 102209, China. }\end{array}$ & 112 & $6,06 \%$ \\
\hline 10 & $\begin{array}{l}\text { State Key Laboratory of Alternate } \\
\text { electrical Power System with } \\
\text { Renewable Energy Sources, North } \\
\text { China Electric Power University, } \\
\text { Beijing, 102206, China. }\end{array}$ & 96 & $5,19 \%$ \\
\hline
\end{tabular}

Fuente: Los autores.

Fig. 8. ORGANIZACIONES CON EL MAYOR NÚMERO DE CITAS EN ARTÍCULOS REFERENTES A LA PLANEACIÓN, EXPANSIÓN DEL SEP A NIVEL MUNDIAL

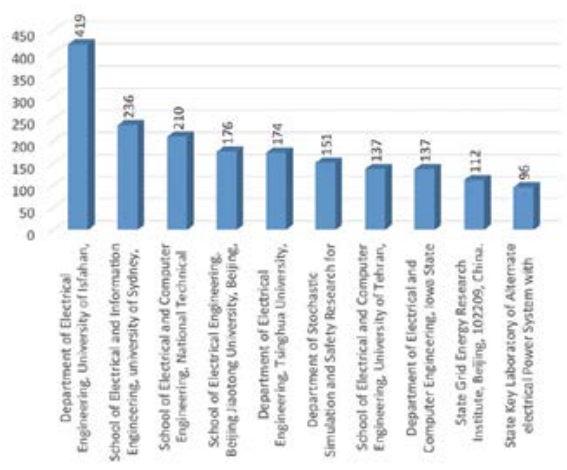

Fuente: Los autores. 


\section{- Elaboración de la matriz del estado del arte}

Después de seleccionar, identificar y depurar los artículos que tienen referencia con el tema investigado, se tuvieron en cuenta 17 artículos para realizar la matriz del estado del arte. Luego de leer y analizar cada uno, se revisaron las temáticas, formulación de problema, restricción del problema, propuestas y soluciones que son planteadas en cada artículo por el/los autor/es, las mismas que se tomarán como base y recomendación para la solución del presente tema de investigación. Toda la información que consta en la matriz del estado del arte es legítima y tiene como respaldo cada artículo publicado; dicha información fue verificada mediante Scholar Google, al ingresar el título del artículo publicado se obtuvo información de la revista donde se publicó, tipo de artículo, número de citas y la fecha de publicación.

Luego de analizar cada artículo en la matriz, se obtuvieron los siguientes resultados, considerados de los artículos más relevantes; la información obtenida es la siguiente. En la Fig. 9 están detalladas las temáticas de mayor importancia del tema investigado y se visualiza que la Expansión corresponde al 22,97\%, la Planificación corresponde al $20.27 \%$, la Generación corresponde al $20.27 \%$, la Transmisión corresponde al $18.92 \%$ y los Sistemas eléctricos de potencia corresponde al $17.57 \%$.

Fig. 9. TEMÁTICAS DE LA INVESTIGACIÓN BASADO EN LA MATRIZ DEL
ESTADO DEL ARTE

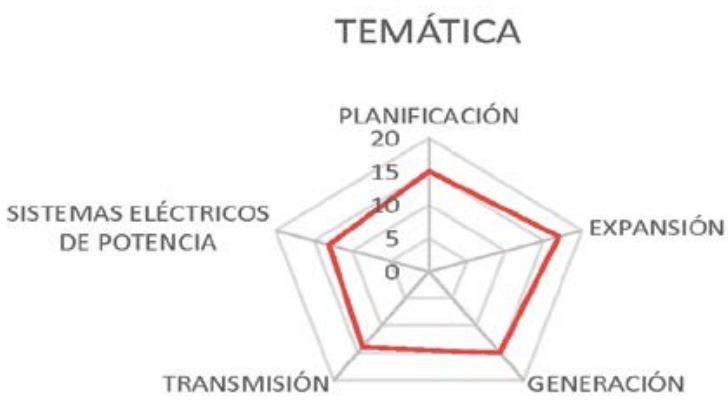

Fuente: Los autores.

En la Tabla V se visualiza la problemática presente en la investigación realizada, y se detalla a continuación: el Crecimiento de la demanda eléctrica corresponde al $19.17 \%$, la Contingencia N-1 corresponde al 12.32\%, la Optimización

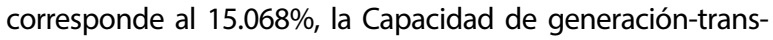
misión corresponde al $16.44 \%$, las Pérdidas en el sistema corresponden al $10.96 \%$, los Costos/Inversión corresponden al $13.69 \%$ y, finalmente, la Confiabilidad corresponde al $12.32 \%$.

Referente a las soluciones propuestas por autores en artículos similares con el presente tema de investigación: "Planificación, expansión del SEP", en la Tabla VI se visualiza que las soluciones propuestas son: MILP corresponde al $22.03 \%$, TEP corresponde al 20.34\%, GEP corresponde al $10.17 \%$, Metodología heurística corresponde al $11.86 \%$,
Algoritmo estocástico corresponde al 13.56\%, Descomposición de Benders corresponde al $16.95 \%$ y Monte Carlo corresponde al $5.08 \%$.

Tabla V.

PROBLEMÁTICA DE LA INVESTIGACIÓN BASADO EN LA MATRIZ DEL ESTADO DEL ARTE

\begin{tabular}{|c|c|c|c|}
\hline $\mathrm{N}^{\circ}$ & Problemática & Ocurrencia & Ponderación \\
\hline 1 & $\begin{array}{c}\text { Crecimiento demanda } \\
\text { eléctrica }\end{array}$ & 14 & $19,17 \%$ \\
\hline 2 & Contingencia N-1 & 9 & $12,32 \%$ \\
\hline 3 & Optimización & 11 & $15,07 \%$ \\
\hline 4 & $\begin{array}{c}\text { Capacidad generación- } \\
\text { transmisión }\end{array}$ & 12 & $16,44 \%$ \\
\hline 5 & Pérdidas en el sistema & 8 & $10,96 \%$ \\
\hline 6 & Costos/Inversión & 10 & $13,69 \%$ \\
\hline 7 & Confiabilidad & 9 & $12,33 \%$ \\
\hline
\end{tabular}

Fuente: Los autores.

Tabla VI. SOLUCIONES PARA LA INVESTIGACIÓN BASADO EN LA MATRIZ DEL ESTADO DEL ARTE

\begin{tabular}{|c|c|c|c|}
\hline $\mathbf{N}^{\circ} 1$ & Solución & Ocurrencia & Ponderación \\
\hline 1 & $\begin{array}{l}\text { MILP Programación Lineal } \\
\text { de Enteros Mixtos }\end{array}$ & 13 & $22,03 \%$ \\
\hline 2 & $\begin{array}{l}\text { TEP Planificación de } \\
\text { la Expansión de la } \\
\text { Transmisión }\end{array}$ & 12 & $20,34 \%$ \\
\hline 3 & $\begin{array}{l}\text { GEP Planificación de } \\
\text { la Expansión de la } \\
\text { Generación }\end{array}$ & 6 & $10,17 \%$ \\
\hline 4 & Metodología Heurística & 7 & $11,86 \%$ \\
\hline 5 & Algoritmo Estocástico & 8 & $13,56 \%$ \\
\hline 6 & $\begin{array}{c}\text { Descomposición de } \\
\text { Benders }\end{array}$ & 10 & $16,95 \%$ \\
\hline 7 & Monte Carlo & 3 & $5,08 \%$ \\
\hline
\end{tabular}

Fuente: Los autores.

\section{CONCLUSIONES}

Basado en lo expuesto en la literatura científica se puede aseverar que existen diferentes metodologías con diferente desempeño que permiten realizar la planificación de la expansión del SEP planteando el GEP y TEP de manera individual y conjunta, considerando el crecimiento de la 
demanda eléctrica y cumpliendo con niveles de confiabilidad y calidad en todo el sistema.

El SEP debe garantizar un continuo funcionamiento, niveles de voltaje, respetar los límites técnicos operativos y considerar la posibilidad de la ocurrencia de contingencias en cualquier elemento del sistema, para ello la literatura considera contingencias $\mathrm{N}-1$ y contingencias $\mathrm{N}-2$ como restricciones de los modelos de TEP.

Las metodologías más empleadas para resolver los problemas de GEP y TEP según la literatura son técnicas de optimización numéricas, técnicas heurístas y metaheurísticas, y técnicas basadas en inteligencia artificial.

La GEP se resuelve utilizando las herramientas MILP de gran escala, métodos heurísticos, algoritmo iterativo de punto fijo, método Karush - Kuhn - Tucker, para sistemas de gran tamaño se emplea CPF como metodología que encuentra la solución a sistemas infactibles por la complejidad de los modelos.

Los flujos óptimos de potencia DC y AC son herramientas utilizadas para la resolución de la expansión del SEP, el investigador debe elegir el adecuado, porque el flujo óptimo AC presenta más complejidad que el DC, pero a la vez nos entrega una solución real del comportamiento del sistema.

El análisis bibliométrico permitió determinar que los países China, EE.UU, Irán en ese orden han aportado con la mayor cantidad de artículos, autores, números de citas y organizaciones.

\section{REFERENCIAS}

[1] M. Mehrtash and A. Kargarian, "Risk-based dynamic generation and transmission expansion planning with propagating effects of contingencies," Int. J. Electr. Power Energy Syst., vol. 118, no. October 2019, p. 105762, 2020. DOI: https://doi.org/10.1016/j.ijepes.2019.105762

[2] D. Carrión, J. Palacios, M. Espinel, and J. González, "Transmission Expansion Planning Considering Grid Topology Changes and N-1 Contingencies Criteria," Recent Advances in Electrical Engineering, Electronics and Energy, 2021, vol. 2, pp. 266-279. DOI: https://doi.org/10.1007/978-3-030-72208-1

[3] A. C. L. de Oliveira, I. M. de Mendonça, F. G. Duque, N. dos S. Renato, and I. C. da Silva Junior, "A new proposal of static expansion planning of electric power transmission systems using statistical indicators," Reliab. Eng. Syst. Saf., vol. 200, no. September 2019, p. 106928, 2020. DOI: https://doi.org/10.1016/j.ress.2020.106928

[4] H. Mavalizadeh et al., "Multiobjective Robust Power System Expansion Planning Considering Generation Units Retirement," IEEE Syst. J., vol. 12, no. 3, pp. 2664-2675, 2017. DOI: https://doi.org/10.1109/JSYST.2017.2672694

[5] Z. Wu, Y. Liu, W. Gu, Y. Wang, and C. Chen, "Contingencyconstrained robust transmission expansion planning under uncertainty," Int. J. Electr. Power Energy Syst., vol. 101, no.
February, pp. 331-338, 2018. DOI: https://doi.org/10.1016/j. ijepes.2018.03.020

[6] R. C. G. Teive, E. L. Silva, and L. G. S. Fonseca, "A cooperative expert system for transmission expansion planning of electrical power systems," IEEE Power Eng. Rev., vol. 13, no. 2, pp. 636-642, 1997. DOl: https://doi.org/10.1109/59.667393

[7] M. Majidi-Qadikolai and R. Baldick, "Stochastic Transmission Capacity Expansion Planning with Special Scenario Selection for Integrating \$n-1\$ Contingency Analysis," IEEE Trans. Power Syst., vol. 31, no. 6, pp. 4901-4912, 2016. DOI: https://doi.org/10.1109/TPWRS.2016.2523998

[8] D. B. Aeggegn, A. O. Salau, and Y. Gebru, "Load flow and contingency analysis for transmission line outage," Arch. Electr. Eng., vol. 69, no. 3, pp. 581-594, 2020. DOI: https://doi.org/10.24425/aee.2020.133919

[9] M. Majidi-qadikolai, S. Member, and R. Baldick, "Integration of N-1 Contingency Analysis With Systematic Transmission Capacity Expansion Planning: ERCOT Case Study," vol. 31, no. 3, pp. 2234-2245, 2015. DOI: https://doi.org/10.1109/TPWRS.2015.2443101

[10] H. A. S. Abushamah, M. R. Haghifam, and T. G. Bolandi, "A novel approach for distributed generation expansion planning considering its added value compared with centralized generation expansion," Sustain. Energy, Grids Networks, vol. 25, p. 100417, 2021. DOI: https://doi.org/10.1016/j.segan.2020.100417

[11] J. Aghaei, N. Amjady, A. Baharvandi, and M. A. Akbari, "Generation and transmission expansion planning: MILP-based probabilistic model," IEEE Trans. Power Syst., vol. 29, no. 4, pp. 1592-1601, 2014. DOI: https://doi.org/10.1109/TPWRS.2013.2296352

[12] D. Do, U. Oh, Y. Lee, J. Choi, and B. Lee, "A Novel Dynamic Index of Voltage Instability Expectation with Power System Contingency," J. Electr. Eng. Technol., vol. 14, no. 4, pp. 14631472, 2019. DOI: https://doi.org/10.1007/s42835-019-00158-1

[13] H. Zhang, G. T. Heydt, V. Vittal, and J. Quintero, "An improved network model for transmission expansion planning considering reactive power and network losses," IEEE Trans. Power Syst., vol. 28, no. 3, pp. 3471-3479, 2013. DOI: https://doi.org/10.1109/TPWRS.2013.2250318

[14] M. Taherkhani, S. H. Hosseini, M. S. Javadi, and J. P. S. Catalão, "Scenario-based probabilistic multi-stage optimization for transmission expansion planning incorporating wind generation integration," Electr. Power Syst. Res., vol. 189, no. February, p. 106601, 2020. DOI: https://doi.org/10.1016/j.epsr.2020.106601

[15] J. Cervantes and F. Fred Choobineh, "A QuantileBased Approach for Transmission Expansion Planning," IEEE Access, vol. 8, pp. 82630-82640, 2020. DOI: https://doi.org/10.1109/ACCESS.2020.2991127

[16] D. Quiroga, E. Sauma, and D. Pozo, “Power system expansion planning under global and local emission mitigation policies," Appl. Energy, vol. 239, pp. 1250-1264, 2019. DOI: https://doi.org/10.1016/j.apenergy.2019.02.001

[17] P. Kongmany, S. Premrudeepreechacharn, and K. Charoenpatcharakij, "Transmission system reliability evaluation in the Central- 1 and Northern regions of the Lao PDR in corresponding to transmission system development plan," Asia-Pacific Power Energy Eng. Conf. APPEEC, 2009. DOI: https://doi.org/10.1109/APPEEC.2009.4918946

[18] P. Masache, D. Carrión, and J. Cárdenas, "Optimal Transmission Line Switching to Improve the Reliability of 
the Power System Considering AC Power Flows," Energies, pp. 1-17, 2021. DOI: https://doi.org/10.3390/en14113281

[19] G. Gutiérrez-Alcaraz, N. González-Cabrera, and E. Gil, "An efficient method for Contingency-Constrained Transmission Expansion Planning," Electr. Power Syst. Res., vol. 182, no. January, p. 106208, 2020. DOI: https://doi.org/10.1016/j.epsr.2020.106208

[20] A. Dini, A. Azarhooshang, S. Pirouzi, M. Norouzi, and M. Lehtonen, "Security-Constrained generation and transmission expansion planning based on optimal bidding in the energy and reserve markets," Electr. Power Syst. Res., vol. 193, no. December 2020, p. 107017, 2021. DOI: https://doi.org/10.1016/j.epsr.2020.107017

[21] A. M. Leite da Silva, L. S. Rezende, L. M. Honorio, and L. A. F. Manso, "Performance comparison of metaheuristics to solve the multi-stage transmission expansion planning problem," IET Gener. Transm. Distrib., vol. 5, no. 3, pp. 360-367, 2011. DOI: https://doi.org/10.1049/iet-gtd.2010.0497

[22] R. Hemmati, R. A. Hooshmand, and A. Khodabakhshian, "Comprehensive review of generation and transmission expansion planning," IET Gener. Transm. Distrib., vol. 7, no. 9, pp. 955-964, 2013. DOI: https://doi.org/10.1049/iet-gtd.2013.0031

[23] J. Palacios and D. Carrión, "Estado del arte de la planeación de expansión de sistemas de transmisión," I+D Tecnológico, vol. 16, no. 2, 2020. DOI: https://doi.org/10.33412/idt.v16.2.2835

[24] P. Freitas, L. Macedo, and R. Romero, "A strategy for transmission network expansion planning considering multiple generation scenarios," Electr. Power Syst. Res., vol. 172, no. December 2018, pp. 22-31, 2019. DOI: https://doi.org/10.1016/j.epsr.2019.02.018

[25] I. M. de Mendonça, I. C. Silva Junior, B. H. Dias, and A. L. M. Marcato, "Identification of relevant routes for static expansion planning of electric power transmission systems," Electr. Power Syst. Res., vol. 140, pp. 769-775, 2016. DOI: https://doi.org/10.1016/j.epsr.2016.05.011

[26] R. O'Neill, E. Krall, K. Hedman, and S. . Oren, “A model and approach to the challenge posed by optimal power systems planning," Math. Program., vol. 140, no. 2, pp. 239-266, 2013. DOI: https://doi.org/10.1007/s10107-013-0695-3

[27] M. Cañas-carretón and M. Carrión, "Generation Capacity Expansion Considering Reserve Probvision by Wind Power Units," IEEE Trans. Power Syst., vol. 35, no. 6, pp. 4564-4573, 2020. DOI: https://doi.org/10.1109/TPWRS.2020.2994173

[28] E. Gil, I. Aravena, and R. Cárdenas, "Generation Capacity Expansion Planning Under Hydro Uncertainty Using
Stochastic Mixed Integer Programming and Scenario Reduction,"IEEE Trans. Power Syst., vol. 30, no. 4, pp. 1838-1847, 2015. DOI: https://doi.org/10.1109/TPWRS.2014.2351374

[29] G. A. Bakirtzis, P. N. Biskas, and V. Chatziathanasiou, "Generation expansion planning by MILP considering midterm scheduling decisions," Electr. Power Syst. Res., vol. 86, pp. 98-112, 2012. DOl: https://doi.org/10.1016/j.epsr.2011.12.008

[30] Y. Feng and S. M. Ryan, "Scenario construction and reduction applied to stochastic power generation expansion planning," Comput. Oper. Res., vol. 40, no. 1, pp. 9-23, 2013. DOI: https://doi.org/10.1016/j.cor.2012.05.005

[31] S. A. Rashidaee, T. Amraee, and M. Fotuhi-Firuzabad, "A linear model for dynamic generation expansion planning considering loss of load probability," IEEE Trans. Power Syst., vol. 33, no. 6, pp. 6924-6934, 2018. DOI: https://doi.org/10.1109/TPWRS.2018.2850822

[32] H. Nguyen and F. Felder, "Generation expansion planning with renewable energy credit markets: A bilevel programming approach," Appl. Energy, vol. 276, no. December 2019, p. 115472, 2020. DOI: https://doi.org/10.1016/j.apenergy.2020.115472

[33] E. de Oliveira, I. da Silva, J. Pereira, and S. Carneiro, "Transmission system expansion planning using a sigmoid function to handle integer investment variables," IEEE Trans. Power Syst., vol. 20, no. 3, pp. 1616-1621, 2005. DOI: https://doi.org/10.1109/TPWRS.2005.852065

[34] R. Mahanty and P. Gupta, "Transmission network expansion planning with security constraints," IEE ProceedingsGeneration, Transm. ..., vol. 151, no. 3, pp. 201-212, 2004. DOI: https://doi.org/10.1049/ip-gtd

[35] I. G. Sardou and E. Azad-Farsani, "Network expansion planning with microgrid aggregators under uncertainty," IET Gener. Transm. Distrib., vol. 12, no. 9, pp. 2105-2114, 2018. DOI: https://doi.org/10.1049/iet-gtd.2017.1076

[36] S. Lumbreras and A. Ramos, "The new challenges to transmission expansion planning. Survey of recent practice and literature review," Electr. Power Syst. Res., vol. 134, pp. 19-29, 2016. DOI: https://doi.org/10.1016/j.epsr.2015.10.013

[37] R. Chen, A. Cohn, N. Fan, and A. Pinar, "Contingencyrisk informed power system design," IEEE Trans. Power Syst., vol. 29, no. 5, pp. 2087-2096, 2014. DOI: https://doi.org/10.1109/TPWRS.2014.2301691 